\title{
Arthroscopic treatment of femoroacetabular impingement in patients older than 60 years
}

\author{
Rodrigo Mardones \\ Alessio Giai Via \\ Alvaro Rivera \\ Alexander Tomic \\ Marcelo Somarriva \\ Mauricio Wainer \\ Daniel Camacho
}

Department of Adult Reconstruction Surgery

Hip/Knee and Hip Arthroscopy,

Clínica Las Condes, Santiago de Chile, Chile

Corresponding author:

Rodrigo Mardones

Head of Department of Adult Reconstruction Surgery

Hip/Knee and Hip Arthroscopy, Clínica Las Condes

La Fontecilla 441, Las Condes, Santiago de Chile,

Chile

E-mail: rmardones@clc.cl

\section{Summary}

Background: The indications of hip arthroscopy increased over the past decade. Although mostly recommended for treatment of femoroacetabular impingement (FAl) in young patients, well-selected older patients ( $>60$ years old) may benefit from this surgery. However, the role of hip arthroscopy for the management of older patients is controversial. The aim of the study is to evaluate the clinical outcomes of a series of patients aged 60 years and older who underwent hip arthroscopy for FAI at mid-term follow-up.

Materials and methods: Sixty-year-old patients and older, with a joint space greater than $2 \mathrm{~mm}$, and a grade I and II hip osteoarthrosis (OA) according Tönnis scale were included into the study. Twentythree patients (28 hips) met the inclusion criteria. The T-Student test was used to detect for differences between variables $(p<0.05)$.

Results: The mean age of the patients was 63.4 years, and the mean follow-up was 4.4 years (2-9 years). We found an improvement in mHHS and VAS score from the baseline to the final follow-up in $87 \%$ of patients $(p<0.05)$. Three patients $(13 \%)$ were submitted to a THA at a mean of 12 months, while the survivorship rate at the final follow-up was $75 \%$. No major complications have been reported.
Conclusion: Arthroscopic treatment of FAl in patients over 60 years old, with no signs of advanced osteoarthrosis, showed a significant improvement of functional score and pain in most of cases, and it can be consider a reasonable option in well selected patients.

Level of evidence: IV case series.

KEY WORDS: hip arthroscopy, femoroacetabular impingement, elderly patients, osteoarthritis.

\section{Introduction}

Hip arthroscopy for the management of femoroacetabular impingement (FAI) increased over the past decade, and indications for surgery have been evolving as our understanding of hip pathology improved. Currently, many intra- and extra-articular pathologies have been recognized as suitable for hip arthroscopy, but even if the indications for this technique have been expanded, its role in the management of patients with mild to moderate osteoarthrosis (OA) is controversial ${ }^{1}$.

Many elderly but still active patients with mild to moderate $\mathrm{OA}$, refer to the clinic with hip pain due to $\mathrm{FAI}$, and even if they may be candidates for hip arthroplasty, they are reluctant to consider joint replacement a suitable option. In these cases counseling may be difficult because of the paucity of literature that pertains to this kind of patients. Although many studies showed that the presence of OA negatively affects the results after hip arthroscopy, with a 16 to $52 \%$ conversion to total hip arthroplasty (THA) ${ }^{2-4}$, recent reports suggest that an improvement in outcomes can be achieved. The selection of patients is of crucial importance in these cases. Philippon et al. ${ }^{5}$ reported that less than $2 \mathrm{~mm}$ joint space preoperatively was predictive of poor outcomes in a cohort of patients who underwent FAl correction at a minimum of 2 years follow-up. More recently, improvement in pain and function have been reported after arthroscopic FAl correction in the presence of mild hip OA (50\% or more than $2 \mathrm{~mm}$ joint space remaining $)^{6}$. Therefore, well-selected elderly patients may benefit from this technique.

The purpose of this study is to evaluate the clinical outcomes of a series of patients aged 60 years and older who underwent hip arthroscopy for FAl treatment at mid-term follow-up. Our hypothesis is that well-selected patients may expect satisfactory results. 


\section{Materials and methods}

We reviewed retrospectively the data of patients who underwent hip arthroscopy for FAI treatment between January 2007 and December 2014. Both surgical and study procedures were performed after the patients had signed a written consent and after approval by the local Internal Review Board (IRB-Clinica Las Condes, Santiago de Chile, Chile). The study was performed according the basic principles and recommendations in clinical and field science research? ${ }^{7}$.

\section{Inclusion and exclusion criteria}

Inclusion criteria were patients aged 60 years and older with symptomatic FAI, patients with a joint space grater than $2 \mathrm{~mm}$, and a grade I and II hip OA according Tönnis scale. Patients with a Tönnis grade greater than II, revision hip arthroscopy, patients with co-morbidities predisposing to osteoarthritis such as hip dysplasia (defined as Wiber angle $<20^{\circ}$ ), rheumatological disorders, avascular necrosis, LeggCalve'-Perthes disease, Ehlers-Danlos syndrome, chronic renal failure, previous intra-articular fractures were excluded. Patients were excluded if they underwent gluteus medius repair. The need of a revision hip arthroscopy, a Modified Harris Hip Score (mHHS) less than $70^{6}$, and the implant of a THA were considered as a failure of the treatment. Twenty-three patients (5 men, 18 women) met the inclusion criteria. Five patients were treated bilaterally, for a total of 28 hips.

\section{Patient assessment}

A detailed physical examination was conducted in all patients, who were examined by an orthopaedic surgeon with a full training and great experience in hip and pelvic surgery (R.M.). The FADIR test (anterior impingement test) was considered positive if pain was elicited in forced flexion combined with internal rotation of the hip. The FABER test was also evaluated in all cases. The hip Range of Motion (ROM) was evaluated and collected. All physical examinations were performed and documented in degrees in a clinical setting. For the imaging analysis, the set of plain radiographs included pelvis anterior-posterior (AP) view, and AP and cross-table view of the affected hip (Figure 1). Assessment of radiographic signs of FAI was performed, and special attention was given to any articular space narrowing. Cam impingement was assessed by the measurement of the a-angle on the cross-table view of the hip and femoral head-neck offset $^{8}$. An a-angle greater than $50^{\circ}$ and a femoral head-neck offset lower than $10 \mathrm{~mm}$ were considered pathologic ${ }^{9}$. Pincer impingement was defined as a lateral center edge angle (LCEA) greater than $35^{\circ}$ by Wiberg, and/or an extrusion index smaller than $20 \%{ }^{10}$. Joint space narrowing was graded by measuring the narrowest distance between the femoral head and acetabulum at the lateral and middle sourcil on weightbearing AP pelvis radiographs ${ }^{10}$. Osteoarthritis was assessed from the AP pelvic and graded according to the Tönnis classification ${ }^{11}$. An MRI arthrogram was performed in all cases, that showed a torn and calcified labrum. At the time of the arthrogram, a lidocaine test (intra-articular shot of lidocaine) was applied, and the result was positive (temporary relief of pain) in all cases. This was performed to make sure of the intra-articular source of pain. The mHHS was administered to all patients before surgery (TO), at 1 year follow-up (T1) and final follow-up (T2). The VAS score was also administered preoperatively and at each control. Follow-up data were obtained through

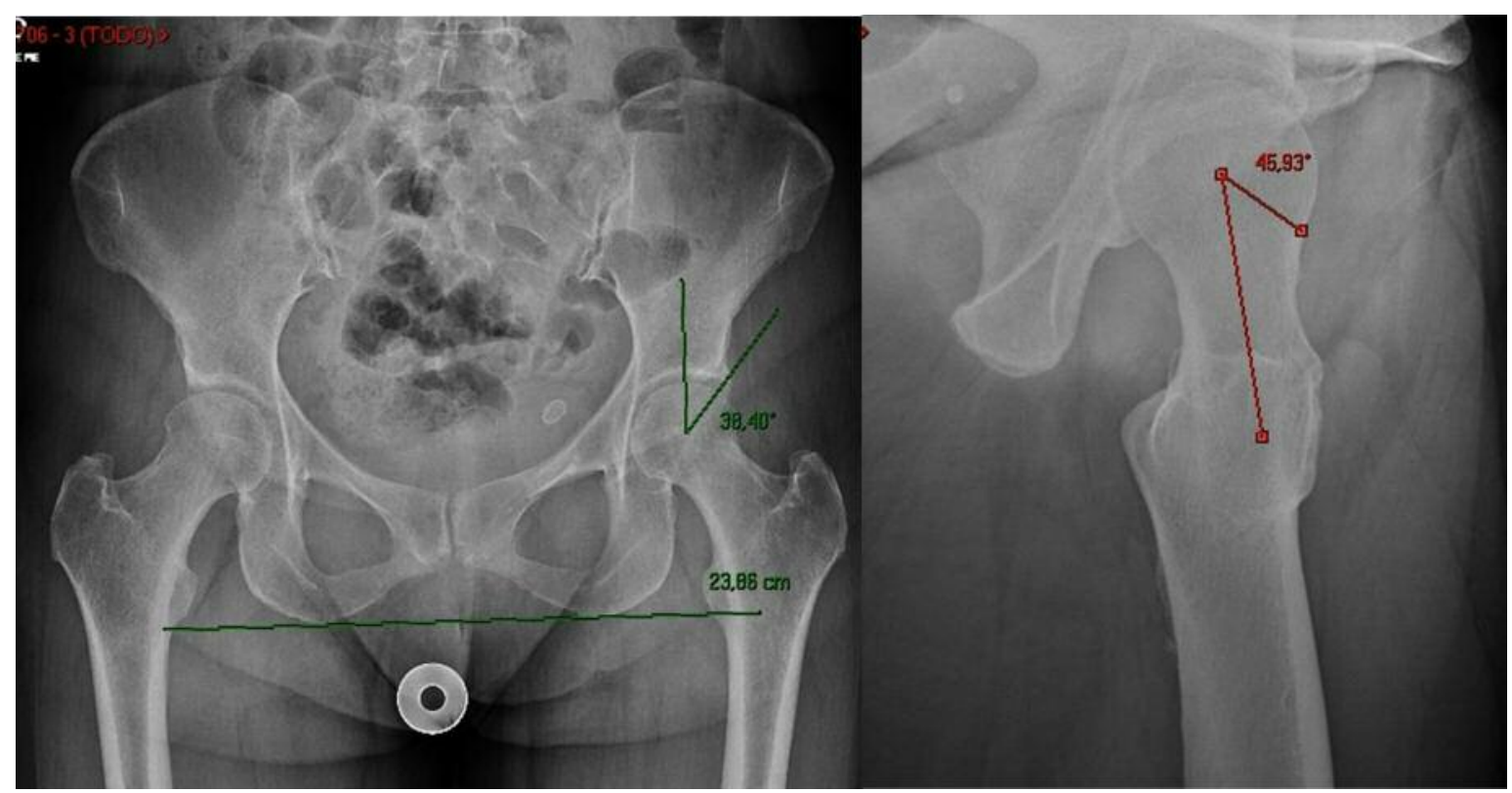

Figure 1. Pre-operatory X-Ray of a 61 years old woman submitted to hip arthroscopy for femoroacetabular impingement correction. 
office visits (R.M.; M.S.; M.W.), and a phone calls from a fellow (A.R.V.). The date of the office visits or of the phone call was used as the follow-up date.

\section{Surgical technique}

The patient was positioned supine on a fracture table. Under combined anesthesia (spinal anesthesia and deep sedation), traction was applied to the affected lower limb and the hip was distracted. To confirmed hip joint distraction an image intensifier was used. Than traction was released, and the hip and the lower limb were prepared and draped in the usual sterile fashion. After that the hip joint was distracted, the anterolateral portal was performed by introducing a fluoroscopy-guided spinal needle in the central compartment, and a second modified anteromedial portal was established under direct vision ${ }^{12}$. A $70^{\circ}$ arthroscope was used. The procedures performed were acetabuloplasty and osteoplasty of the femoral head-neck junction. The labrum was debrided and repaired with bonny anchors. Microfractures were performed at the labrum-cartilage junction in case of chondral lesions. A functional test of hip motion under direct arthroscopic visualization to rule out any abnormal contact between the femoral head-neck junction and the acetabular rim was performed at the end of the procedure as well. Platelet Rich Plasma (PRP) was positioned at the head-neck junction in all cases. The skin was closed with no-absorbable sutures. Post-operatively, all patients underwent supine standard X-ray of the pelvis and cross-table view of the hip (Figure 2).

The rehabilitation program included walking with 2 crutches as tolerated from the first day after surgery. Patients were encouraged to walk with one crutch beginning the $4^{\text {th }}$ week and to walk without any assis- tance devices beginning the $6^{\text {th }}$ week as tolerated. Full weightbearing was progressively achieved. Continuous passive motion device was used for 12 hours starting immediately after the surgery. Stationary bicycle was also indicated beginning the day after surgery to prevent capsular adhesions and joint stiffness. Celecoxib $200 \mathrm{mg}$ twice a day was prescribed for 3 weeks as prophylaxis of heterotopic calcifications.

\section{Statistical analysis}

Descriptive statistics are presented as mean $( \pm S D)$. Before each statistical analysis, the normal distribution of the population was tested with Shapiro-Wilk test and the homogeneity of the variance was verified with Bartlett's test. For each variable (mHHS and VAS) a student's t-test for independent samples was used to detect any significantly differences. A significance level was set at $p<0.05$. The Statistical analysis was performed with SigmaPlot 11.0 software (Systat Software, Tulsa, OK, USA).

\section{Results}

The mean age of patients was 64.3 years (range 6081 ; S.D. 5.1), and the mean follow-up was 4.4 years (range 2-9.1 years; SD: 2.1 years).

Based on preoperative plain radiographs, the Tönnis grade was 1 in 17 hips, 2 in 11 hips. At the end of the follow-up, we found an evolution of the hip osteoarthrosis grade in 6 hips.

We found a statistically significant improvement of the mHHS between baseline, T1 and T2. The median preoperative $\mathrm{mHHS}$ was 53 (range, 38-66; $\mathrm{SD}=8.4$ ) and improved to 78 (range, 44-98; $\mathrm{SD}=12.8$ ) at 1 year

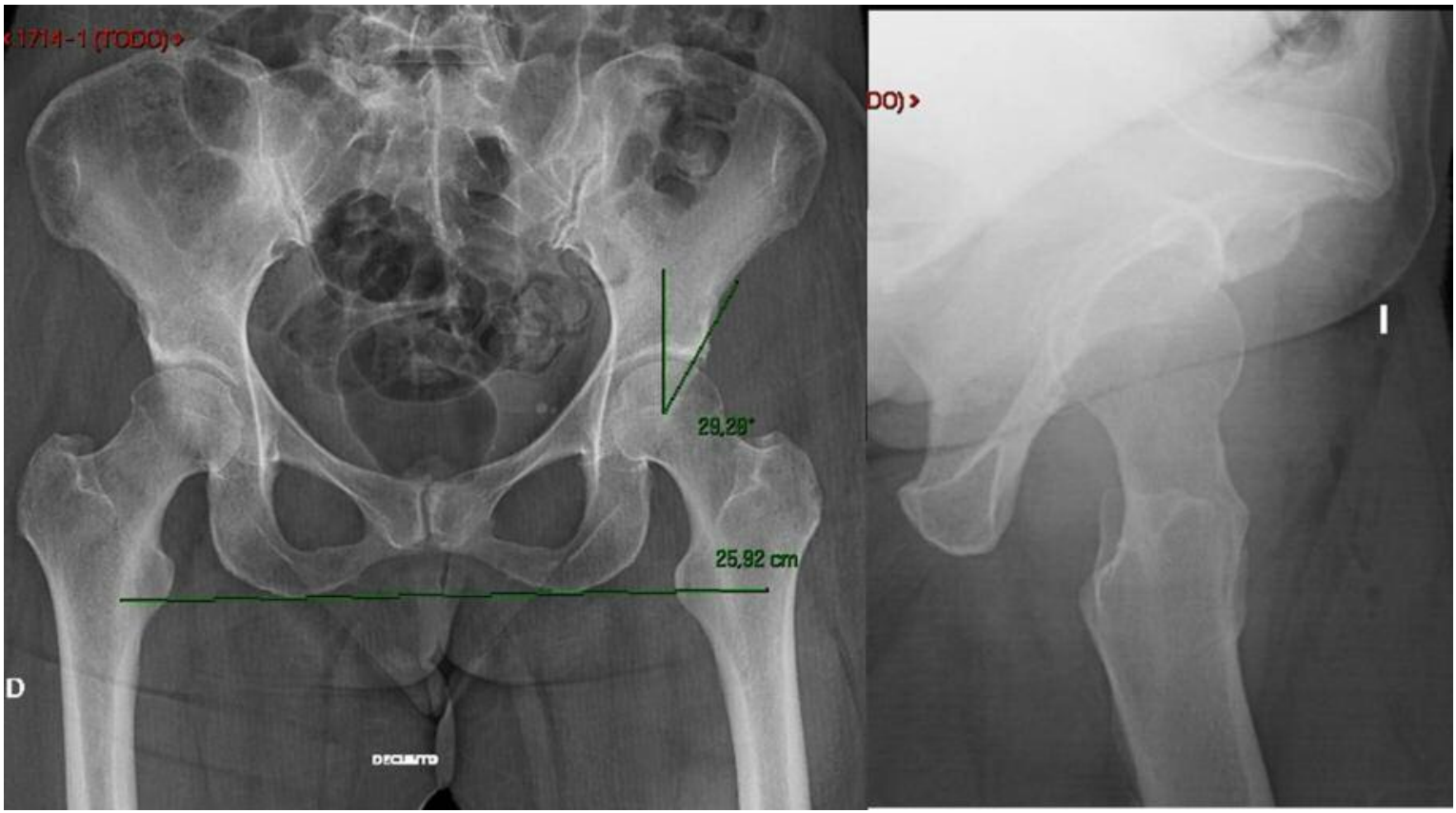

Figure 2. Post-operatory X-Ray of the same patient. 
follow-up $(p<0.05)$. At final follow-up the median mHHS remained 78 (range, 40-92; SD=15.3), without any statistically significant difference compared to 1 year follow-up, but significantly improved compared to T0. The VAS score also improved from a median of 6 preoperatively (range, $4-9 ; S D=1.2$ ) to 2 (range, 0 5 ; $S D=1.1)$ at 1 year follow-up $(p<0.05)$. At last follow the median VAS score was 1 (range, $0-3 ; S D=1.0$ ), and the difference was statistically significant compared to T1 $(p=0.04)$. We found also an improvement of $10^{\circ}$ of internal rotation in most of patients, and of $5^{\circ}$ with the hip at $90^{\circ}$ of flexion, but the difference was not statistically significant.

No patients need a revision hip arthroscopy during the follow-up period, 4 patients had a mHHS less than 70 at final follow-up, and 3 patients were submitted to a THA (13\% of the hips), at the mean of 12 months post intervention. Two of these patients had a coxa profunda and a preoperatory Tönnis grade of $\mathrm{I}$, and the other hip had a preoperatory Tönnis grade of II. Therefore, the failure rate at final follow-up was $25 \%$. Neither major complications nor infections have been reported in this court of patients.

\section{Discussion}

Hip arthroscopy is a minimally invasive technique with a growing demand. The current indications are numerous and will probably expand over time. Although mostly recommended for young people with an overall good articular status, well selected older patients ( $>60$ years old) may benefit from this surgery ${ }^{13}$. The number of active elderly patients who refer to orthopaedic surgeons with intra-articular hip pain is growing. They are usually inclined to continue their sport activity and to preserve their hip joint as long as possible. They are looking for a preserving treatment and they are reluctant to consider the idea of a joint replacement, even if a THA would be a proper indication. Results of hip arthroscopy in patients affected by FAl and associated $O A$ are controversial. Many studies suggested that outcomes in patients affected by articular cartilage injuries are poor, with an high rate of failure ${ }^{14}$. Daivajna et al. reported a conversion rate to THA of $43 \%$ in 77 patients with a Tönnis grade II and III hip $\mathrm{OA}$ at a mean of 18 months follow-up, concluding that in these cases a primary hip arthroplasty should be preferred $^{15}$. However, recent articles reported encouraging results in well selected cases.

A careful selection of patients is of extremely importance. Philippon et. $\mathrm{al}^{16}$ stated that patients with a joint space smaller than $2 \mathrm{~mm}$ who undergo arthroscopic surgery have an higher risk of early conversion to THA. The degree of joint space narrowing is an independent predictor for poor outcomes and high failure rates. Larson et al. ${ }^{6}$ reported than patients without significant preoperative joint space narrowing or a joint space greater than $2 \mathrm{~mm}$ had improved scores and lower failure rate compared to patients with an advanced joint space narrowing (33 vs $82 \%$ of failure rate). Furthermore, even if patients with preoperative radiographic joint space narrowing had improved mean $\mathrm{HHS}$ at 1 year postoperatively compared with preoperative scores, they had no improvements at longer follow-up. The Authors also found that a greater duration of symptoms before surgery was another independent predictor of failure. If age is a predictor factor of poor outcomes is still debated. While Margheritini and Villar ${ }^{17}$ found that greater age was predictive of a poor HHS for patients with OA undergoing hip arthroscopy, other Authors did not find this association ${ }^{6}$. Other risk factors for conversion to THA may be low preoperative $\mathrm{mHHS}$ (lower than 50), high preoperative VAS score (greater than 7), and evidence of hip dysplasia, with a greater than 2 -fold risk of early conversion to $\mathrm{THA}^{18}$.

In this study, we found that hip arthroscopy in well selected elderly patients may improve outcomes and function at a middle-term follow-up. A study by Jerosch et al. ${ }^{19}$ showed a significant improvement in terms of function in 18 of 22 patients with early and midstage OA. Recently Redmond et al. ${ }^{18}$ reported on 32 patients aged 60 years and older, with mean follow-up of 2.5 year. They found a survivorship rate of $70 \%$ at 2 year follow-up, with a good improvement in functional scores and pain, and a conversion rate to THA of $30 \%$ at a mean of 1.1 years. The Authors concluded that age was not a risk factor for failure, but arthroscopic treatment in elderly patients should be approached with caution. Larson et al. ${ }^{6}$ compared arthroscopic treatment of $\mathrm{FAl}$ in patients with no joint space narrowing (FAl group) to patients with slight joint space narrowing (less than $2 \mathrm{~mm}$ ) and patients with advanced joint space narrowing and OA (greater than $2 \mathrm{~mm}$; FAI-OA group). The Authors found an improvement in HHS and VAS score in the FAI-group and in patients with slight joint space narrowing, which was maintained during the study period, while no improvements were found in the FAI-OA group. The conversion rate to THA was $0.6 \%$ in patients without joint space narrowing, $22 \%$ in patients with a slight joint space narrowing, and $57 \%$ in patients with a joint space less than $2 \mathrm{~mm}$ at 2 years follow-up. The overall failure rate at last follow-up (3 years) was $12 \%$ for the FAI-Group, 33\% for patients with slight preoperative joint space narrowing, and $82 \%$ for $\mathrm{FAl}-$ OA group. In a previous study with a smaller number of patients (15) and shorter follow-up (2 years), the Authors of the present article reported good results in most of patients, with a failure rate (THA) of $20 \%{ }^{13}$.

According to the present study, we found an improvement of functional score and pain in $87 \%$ of our court of patients, which was maintained until the final follow-up. The conversion rate to THA was $13 \%$, while the overall failure rate was $25 \%$. In conclusion, $75 \%$ of the patients had a mHHS greater than 70 at final follow-up and did not require a THA. These results are slightly better than that reported in recent literature, probably because of the careful preoperatory indications. We have estimated that no more than $10 \%$ of the patients older than 60 years old, who refer to our center with hip pain and some grade of hip osteoarthritis may be candidates for hip arthroscopy for the treatment of their underlying FAI. 
We are aware of the limitation of this study. One of the most important is the lack of control group treated conservatively, and we cannot truly evaluate the effectiveness of surgery in comparison to conservative management. However, conservative treatment options failed or had been exhausted in all of the patients of the study. Then, the relatively small sample size is a limitation of the study, making statistical analysis of the data difficult. Evaluation of outcome after hip arthroscopy is also challenged by the lack of dedicated subjective outcome instruments for patients submitted to hip arthroscopy. It is also likely that with longer follow-up, outcomes in patients with established OA will continue to deteriorate. More studies addressing such limitations are necessary to elucidate the real significance of the arthroscopic treatment of $\mathrm{FAl}$ in the presence of OA. New bearing surfaces and techniques regarding total hip replacement are promising. A well-designed study comparing their results with those obtained after hip arthroscopy in this group of patients would be desirable.

\section{Conclusion}

Arthroscopic treatment of $\mathrm{FAl}$ in patients over 60 years old, with no signs of advanced osteoarthritis, showed a significant improvement of functional score and pain in $75 \%$ of cases at middle-term follow-up. The selection of the patient is of extremely importance, and all patients should be counseled before surgery on the possibility of the conversion to THA. Based on this study, we consider this procedure a reasonable option in well selected cases.

\section{Conflict of interests}

The Authors declared no potential conflicts of interest with respect to the Authorship and/or publication of this article.

\section{References}

1. Wilkin G, March G, Beaule PE. Arthroscopic acetabular labral debridement in patients forty-five years of age or older has minimal benefit for pain and function. J Bone Joint Surg Am. 2014:96:113-118.

2. McCarthy JC, Jarrett BT, Ojeifo O, Lee JA, Bragdon CR. What factors influence long-term survivorship after hip arthroscopy?
Clin Orthop Relat Res. 2011;469:362-371.

3. Kim KC, Hwang DS, Lee CH, Kwon ST. Influence of femoroacetabular impingement on results of hip arthroscopy in patients with early osteoarthritis. Clin Orthop Relat Res. 2007;456:128132.

4. Haviv B, O'Donnell J. The incidence of total hip arthroplasty after hip arthroscopy in osteoarthritic patients. Sports Med Arthrosc Rehabil Ther Technol. 2010;2:18.

5. Philippon MJ, Briggs KK, Yen YM, Kuppersmith DA. Outcomes following hip arthroscopy for femoroacetabular impingement with associated chondrolabral dysfunction: minimum two-year follow-up. J Bone Joint Surg Br. 2009;91:16-23.

6. Larson CM, Giveans MR,Taylor M. Does Arthroscopic FAI Correction Improve Function with Radiographic Arthritis? Clin Orthop Relat Res. 2011;469:1667-1676.

7. Padulo J, Oliva F, Frizziero A, Maffulli N. Muscles, Ligaments and Tendons Journal. Basic principles and recommendations in clinical and field science research: 2016 Update. MLTJ. 2016;6(1):1-5.

8. Barton C, Salineros M, Rakhra KS, Beaule' PE. Validity of the Alpha Angle Measurement on Plain Radiographs in the Evaluation of Cam-type Femoroacetabular Impingement. Clin Orthop Relat Res. 2011;469:464-469.

9. Nemtala F, Mardones R, Tomic A. Anterior and Posterior Femoral Head-Neck Offset Ratio in the Cam Impingement. Cartilage. 2010;1:238-241.

10. Tannast M, Siebenrock KA, Anderson SE. Femoroacetabular Impingement: Radiographic Diagnosis-What the Radiologist Should Know. AJR. 2007;188:1540-1552.

11. Tonnis D. Normal values of the hip joint for the evaluation of Xrays in children and adults. Clin Orthop Relat Res. 1976; 119:39-47.

12. Byrd T. Modifed Anterior Portal for Hip Arthroscopy. Arthroscopy Tech. 2013;2:337-339.

13. Mardones R, Nemtala F, Tomic A. Arthroscopic Treatment of Femoroacetabular Impingement in Patients over 60 Years Old: Preliminary Report of a Pilot Study. Cartilage. 2010; 1:188-193.

14. Haviv B, Singh PJ, Takla A, O'Donnell J. Arthroscopic femoral osteochondroplasty for cam lesions with isolated acetabular chondral damage. J Bone Joint Surg Br. 2010;92:629-633.

15. Daivajna S, Bajwa A, Villar R. Outcome of Arthroscopy in Patients with Advanced Osteoarthritis of the Hip. PLoS ONE. 2015;10(1):e0113970.

16. Philippon MJ, Schroder e Souza BG, Briggs KK. Hip arthroscopy for femoroacetabular impingement in patients aged 50 years or older. Arthroscopy. 2012;28:59-65.

17. Margheritini F, Villar RN. The efficacy of arthroscopy in the treatment of hip osteoarthritis. Chir Organi Mov. 1999;84:257261.

18. Redmond JM, Gupta A, Cregar WM, Hammarstedt JE, Gui C, Domb BG. Arthroscopic Treatment of Labral Tears in Patients Aged 60 Years or Older. Arthroscopy. 2015;31:1921-7.

19. Jerosch J, Shunck J, Khoja A. Arthroscopic treatment of the hip in early and midstage degenerative joint disease. Knee Surg Sports Traumatol Arthrosc. 2006;14:641-5. 